\title{
Piper Betle Leaves Extract Works Synergistically with 5-Fluorouracil in Controlling Colorectal Cancer Cell Growth
}

\author{
Pek Leng Ng${ }^{1}$, Hui-min $\mathrm{Neoh}^{2}$, Sue Mian Then ${ }^{2}$, Yasmin Anum Mohd Yusof ${ }^{3}$, Wan Zurinah Wan \\ $\mathrm{Ngah}^{2,3}$, Nor Fadilah Rejab ${ }^{1}$, Kar Yong Pin ${ }^{4}$ and Mee Lee Looi ${ }^{2,5}$ \\ ${ }^{I}$ Department of Biomedical Sciences, Faculty of Allied Health; ${ }^{2}$ UKM Medical Molecular Biology Institute (UMBI); \\ ${ }^{3}$ Department of Biochemistry, Faculty of Medicine, Universiti Kebangsaan Malaysia. ${ }^{4}$ Forest Research Institute \\ Malaysia (FRIM), Kepong. ${ }^{5}$ Taylor's University Lakeside Campus, Subang Jaya
}

Background: Piper betle (PB) leaves (daun sirih in Malay language) extract has been demonstrated to have anticarcinogenic activities in the experimental systems. It has been found the PB leaves extract could enhance the cytotoxic effect of other anticancer drugs in cancer cells. The aim of this study is to investigate the combination effect of PB and 5-fluorouracil (5-FU) in enhancing the cytotoxic potential using colon cancer cell lines.

Methods: We first examined the effect of PB leaves extract on colon cancer cells. Next, we examined whether PB leaves extract could increase the sensitivity of the cells to 5-FU. Isobologram analysis was carried out to elucidate PB-5FU interaction. Apoptotic features of the treated cells were then revealed by Annexin V/ PI stain. HPLC was performed to exclude any possible chemical interaction between the compounds.

Result: IC50 of 5-FU treated HT 29 and HCT 116 was $130.0 \mu \mathrm{M}$ and $12.5 \mu \mathrm{M}$, respectively at 72 hours. IC50 of PB-treated HT $29(200.0 \mu \mathrm{g} / \mathrm{ml})$ and HCT 116 cells $(187.5 \mu \mathrm{g} / \mathrm{ml})$ was observed after 36 hours of treatment. In the presence of PB extract, the cytotoxic effect of 5-FU was observed at a lower dose $(12.5 \mu \mathrm{M})$ and a shorter time (24 hours). HT 29 and HCT 116 cells treated with 5-FU or PB alone induced a greater apoptosis effect compared to the combination treatment. Isobologram analysis indicated PB and 5-FU interacted synergistically in HT 29 cells, but antagonistically in HCT 116 cells. Mixture of 5-FU and PB sample did not show any interaction revealed by HPLC separation.

Conclusion: In the presence of $\mathrm{PB}$, a lower dosage of 5-FU is required to achieve the maximum drug effect. $\mathrm{PB}$ works synergistically with 5-FU in controlling cancer cell growth. However our current result showed this interaction may not solely contribute to the apoptosis pathway. Further investigation should be performed to elucidate the possible mechanism. 